\title{
Exploratory RCT of art therapy as an adjunctive treatment in schizophrenia
}

\author{
PHIL RICHARDSON ${ }^{1}$, KEVIN JONES ${ }^{2}$, CHRIS EVANS ${ }^{3}$, \\ PETER STEVENS ${ }^{4}$, \& ANNA ROWE ${ }^{4}$ \\ ${ }^{1}$ Tavistock $\mathcal{E}$ Portman NHS Trust and University of Essex (formerly United Medical Dental Schools, \\ London University), ${ }^{2}$ Goldsmiths College, London University, ${ }^{3}$ Rampton Hospital, Nottinghamshire \\ NHS Trust and Tavistock \& Portman NHS Trust, and ${ }^{4}$ South London and Maudsley NHS Trust
}

\begin{abstract}
Background: There is no high quality controlled trial evidence for the effectiveness of art therapy in the adjunctive treatment of schizophrenia.

Aims: To conduct the first exploratory RCT of group interactive art therapy (AT) as an adjunctive treatment in chronic schizophrenia.

Method: The outcomes of 43 patients randomized to 12 sessions of AT were compared with those of 47 who received standard psychiatric care. Patients were assessed on a range of measures of symptoms, social functioning and quality of life at pre- and post-treatment and six-month follow-up.

Results: Art therapy produced a statistically significant positive effect on negative symptoms (assessed by Scale for the Assessment of Negative Symptoms) though had little and non-significant impact on other measures.

Conclusions: Limitations of the study included insufficient statistical power and a sub-optimal level of treatment. Nevertheless, the results were sufficiently promising to justify further research along these lines.

Declaration of interest: This research was funded by a grant from the London Region NHS Executive Research and Development Directorate.
\end{abstract}

Keywords: Art therapy, schizophrenia, randomized trial

\section{Background}

Art therapy (AT) is a form of psychotherapy where the process of making images plays a central role in the context of the psychotherapeutic relationship. It has been widely applied to the treatment of patients and service users with a range of mental health problems and across all spectra of severity. It can be delivered to all age groups in a variety of formats including those of individual, group, couple and family therapy; and has been applied as both a short-term and a long-term intervention (Waller \& Gilroy, 1992; Waller, 1993).

AT may be particularly well suited to the needs of service users who find difficulty in expressing verbally, in a useful and non-destructive way, their problematic feelings and life experiences - as is frequently the case among the severely mentally ill. Its nonverbal aspects 
may make it highly relevant to service users with differing language and ethnic backgrounds. As an adjunct to the work of community mental health teams (CMHT) it may offer a potential means both to improve the quality of care of service users with long term mental health problems and to enhance clinical outcome.

There have been a number of promising single case research reports of the effects of AT for this user group (Waller \& Gilroy, 1992). In addition a number of uncontrolled trials of AT have reported encouraging outcomes (e.g., Borchers, 1985; Tibbetts \& Stone, 1990; Potocky, 1993; Saunders \& Saunders, 2000). It is widely agreed, however, that a causal association between a treatment and its apparent outcome cannot be established without the methodological rigour of a randomized controlled trial (Pocock, 1984).

Few such trials have been reported for the use of AT with an adult psychiatric population. Examples include the work of Green and colleagues who evaluated a 10-week art therapy group with chronic psychiatric outpatients and reported significant improvements on a number of self- and therapist-rated indices (Green et al., 1987). In addition Grodner et al. (1982) have reported positive outcomes from a controlled trial of art/movement therapy with schizophrenics and depressives. Similarly Schut et al. (1996) obtained positive results on GHQ scores from a cross-modality grief therapy programme which included a significant art therapy component.

Promising though these studies may appear they suffer shortcomings which limit their external validity. These include lack of follow-up (Green \& Grodner studies); the use of outcome measures of doubtful clinical relevance (e.g., Grodner's outcomes are assessed in terms of change on a transient measure of mood); and confounding of therapy effects: in both the Grodner and Schut studies, AT is one element of a multicomponent treatment and its effects cannot be disentangled from those of the other component approaches to movement therapy and behaviour therapy respectively. Where schizophrenia is concerned, a recent Cochrane review underlined the paucity of controlled trial evidence and called for further research in this area (Ruddy \& Milnes, 2003).

In summary, despite its widespread use there would appear to be little high quality evidence for the efficacy of AT with adult psychiatric patients with severe and/or complex problems. The present article describes a randomized controlled trial to assess the incremental benefits of 12 AT sessions combined with CMHT-based standard psychiatric care (SPC) over SPC alone for people with chronic schizophrenia. It was carried out within a mental health NHS Trust serving a deprived inner city catchment area with a high ethnic minority population and higher than average CPA rates. It should be noted that 12 sessions was regarded as an absolute minimum clinical requirement, though clearly this would be regarded as sub optimal by many art therapists.

\section{Participants and methods}

Ethical approval for the study was obtained from the NHS Mental Health Trust in which it was carried out. The study was designed to conform to the CONSORT2 standards for the conduct of randomized controlled trials (Moher, Schulz, \& Altman, 2001).

Participants were mental health service users with a diagnosis of chronic schizophrenia in active contact with CMHTs within an inner city mental health NHS trust at the time of the study. All CMHTs within the trust were invited to refer patients with a diagnosis of chronic schizophrenia of at least two years duration and excluding those: (i) with organic illness, (ii) with a prior referral to AT services in the previous 2 years, (iii) currently receiving another formal psychological treatment, or (iv) currently admitted to inpatient care. Power calculations, using the GPower software (Faul \& Erdfelder, 1992), and based on the 
expectation of small to medium effect sizes on the main outcome variables, identified a necessary sample size of 64 participants per group with alpha set at .05 and power of 8 .

Following initial screening and informed written consent, patients were randomized to either the ATC or SPC arms of the study. Randomization was conducted using the minimization procedure to limit variation between the treatment arms on: CPA level, chronicity (time since first inception into local service), gender and ethnicity.

\section{Therapy}

Standard Psychiatric Care (SPC) was the regular contact with the CMHT CPN (frequency being determined by CPA level), regular medication review and CPA review meetings. Patients had access to a variety of psychiatric day treatment facilities, which varied according to the local sector facilities and arrangements.

Art therapy, which was added to SPC for the intervention arm of the study, consisted of brief group interactive art therapy involving 12 weekly sessions of one and a half hours. It was conducted according to the guidelines set out in Waller (1993, pp. 22-34). Through the availability and use of art material and associated imagery the therapist promotes a climate in which the service user can learn about and understand those patterns of behaviour which are causing distress. Psychotic patients typically have difficulty in relating to people and in trusting the therapist. Here the specific presence of the image as a crucial part of art therapy can triangulate and temper problematic feelings, e.g., paranoid suspicion, of the patient toward the therapist. This can allow the patient to experience difficult and conflicting feelings as non-threatening; can reduce the tendency to dropout by providing a form of containment of psychotic fears; and can facilitate engagement in psychological treatment.

\section{Assessments}

All participants in the trial were assessed: (i) prior to the first group AT session, (ii) no later than a fortnight following the final group AT session, and (iii) at 6 month follow up. A research assistant who had no involvement in therapy groups and was not aware of the arm to which the participant had been allocated conducted assessments. Since assessments involved interviewing users it is highly improbable that the research assistant could remain completely blind to group allocation. In addition to this research assessment, corroborative information relevant to the main areas assessed was obtained from the CPN or other care co-ordinator. The research assistant received formal training in the use of the measures, which included:

- General socio-demographic, clinical and health care utilization information pertaining to age, gender, ethnicity, marital/living status, socio-economic status, diagnosis, CPA status, chronicity, previous psychiatric history, medication, other current treatments, use of services (primary and secondary care including CPN contacts) using a local standard schedule.

- HONOS Scales (Wing et al., 1998) rated in collaboration with the CPN.

- Brief Psychiatric Rating Scale (BPRS: Overall \& Gorham, 1962) - for observer rated symptoms.

- Social Functioning Scale (SFS: Birchwood et al., 1990) - yielding scores on social withdrawal, interpersonal functioning, prosocial activities, recreation, level of independence, competence, performance and employment. 
- Inventory of Interpersonal Problems (IIP-32: Barkham et al., 1995).

- Scale for the Assessment of Negative Symptoms (SANS: Andreason, 1989) - to assess the major negative symptoms of schizophrenia.

- Lancashire Quality of Life Profile (Perc QoL: Oliver et al., 1996) - designed specifically for long term mental illness.

- Brief Symptom Inventory (BSI: Derogatis \& Melisaratos, 1983) - self rated symptoms.

\section{Statistical methods}

As noted, the design, through the CONSORT2 guidelines, was of a pragmatic RCT and, given the size and the lack of pre-existing RCTs in the area, was explicitly intended to pilot feasibility and estimate approximate effects for a minimal intervention. Hence a single primary outcome measure was not specified (NB this exploratory approach was the basis on which the protocol was accepted by the funding body). Exploratory $95 \%$ confidence interval presentation of results complements inferential tests wherever possible. By prior design, the inferential test of main effects was by analysis of covariance (ANCOVA) of outcome scores with baseline scores as covariates.

\section{Results}

\section{Recruitment and randomization}

Figure 1 shows the flow of recruitment to the trial. It will be noted that, although a total of 452 patients were identified by CMHTs as potential recruits, the final recruitment figure was only 90 , representing $20 \%$ of the original cohort. The major losses to recruitment resulted from meeting exclusion criteria $(10.8 \%, n=49)$ - the commonest being current inpatient status $(n=24)$; failing twice to respond to an invitation to the screening assessment $(22.3 \%, n=101)$; and refusal to consent to participate $(45.6 \%, n=206)$.

After the randomization, which produced 43 participants in the AT arm and 47 in the SPC, the two arms of the trial were near perfectly balanced for gender with 31 men of the 47 in the SPC arm and 28 of the 43 in the AT arm $(66 \%$ cf. $65.1 \% ; 95 \%$ CI diff. -18 to $+20 \%$; exact $p=1.0$ ). The groups did not differ significantly on age: SPC mean 42.6 , SD 11.5, AT mean 39.6, SD 10.5 ; $95 \%$ CI diff. -1.6 to $7.6 ; t(88)=1.3, p=.2$, M-W $\mathrm{U}=857.5, p=.22$. For ethnicity there were no overall differences in distribution between the two arms of the trial (Fisher's exact test extended to tables larger than 2-by-2, $p=.13$ ). Chronicity of problems ranged from 1 to 37 (years). The two groups did not differ in chronicity (SPC mean 13.4, median 10, iqr 7 to 21; AT mean 12.6, median 9, iqr 5 to 22; Mann-Whitney $\mathrm{U}=936, p=.55)$. CPA level, not known for one patient, was not distributed differently between the two arms (exact $p=.75$, Mann-Whitney $\mathrm{U}=937, p=.64$ ). Finally, there was no association of arm with any of: referring consultant (exact $p=.90$ ); postcode $(p=.82)$ or research interviewer $(p=.67)$.

Pre-treatment differences between the two arms were not statistically significant for the IIP $(\mathrm{U}=858, p=.57 ; t(84)=.67, p=.51)$; the BSI $(\mathrm{U}=795.5, p=.84)$; HONOS total $(t(87)=.77, p=.44,95 \% \mathrm{CI}$ diff from -1.3 to +3.1 ; BPRS total $(t(87)=.46, p=.64 ; 95 \%$ CI from -2.8 to +4.6$)$; LQoL total $(\mathrm{U}=833, p=.2 ; t(83.6)=1.2, p=.23,95 \%$ CI diff from -.51 to +.12$)$; or SFS overall score $t(85)=1.1, p=.26$; $95 \%$ CI diff from -12.6 to +3.4 ). For the SANS there was a narrowly significant difference between the arms on baseline scores: SPC mean 9.4, SD 5.2 cf. AT mean 7.4, SD 3.7, Levene $\mathrm{F}=9, p=.004$, $t(81.1)=2.09, p=.04,95 \%$ CI diff from .09 to 3.85 . 


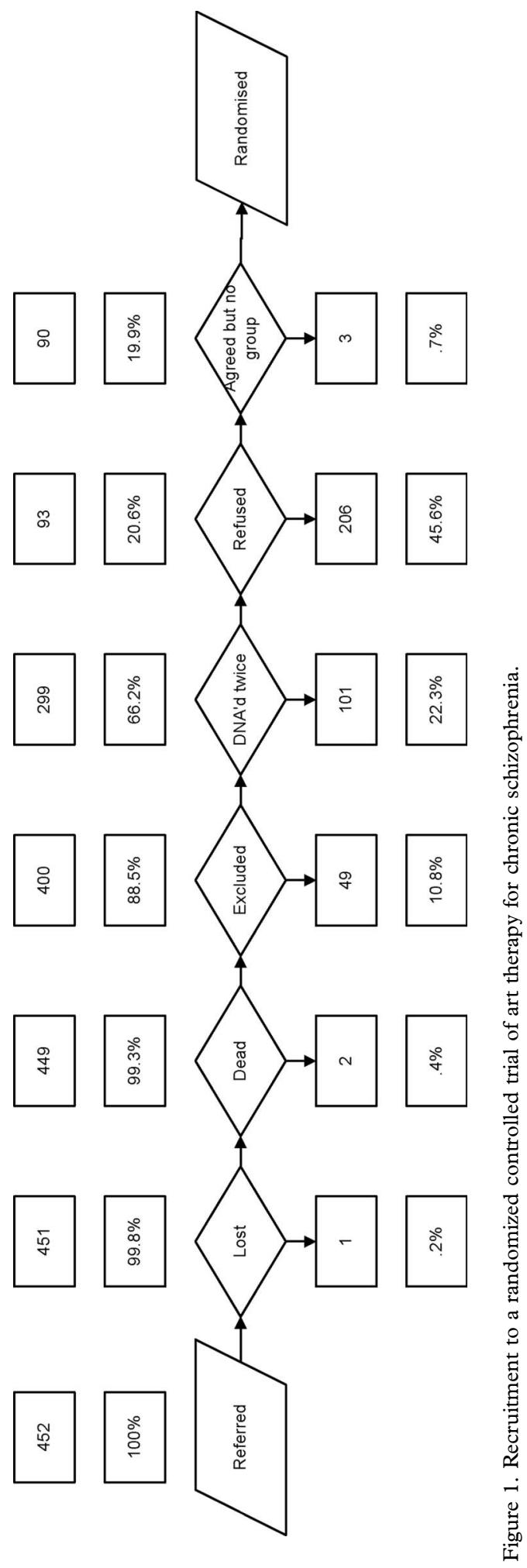




\section{Designed analyses}

Seventy-four (82\%) participants were successfully interviewed within two weeks of completion of the therapy (for the AT arm, at the same time for the SPC arm). Forty $(44 \%)$ were successfully interviewed at six-month follow-up. The designed analyses mostly reveal non-significant differences between the two arms as shown in Table I. However, a statistically significant difference is found both at end of therapy and follow-up for the SANS.

Means and confidence intervals for change and for differences are shown in Table II. The general pattern is of rather little change over time in either arm with confidence intervals for change excluding zero only for SNS at follow-up in the SPC arm (a deterioration over time) and none excluding zero for the AT arm (though for BSI and IIP at end of therapy and for BSI at follow-up the upper end of the interval is at zero). The statistically significant difference between the arms can be seen to be in the direction of greater improvement in the AT arm, though the simple confidence interval for the difference in change only excludes zero at follow-up, not at end of therapy.

\section{Discussion}

The results of the present exploratory study, the first randomized controlled trial of group interactive art therapy for people with schizophrenia, provide only qualified support. On 6 out of 7 outcome measures statistically non-significant differences were found between the treatment and control group patients following treatment. Only on the Scale for the Assessment of Negative Symptoms was a significant incremental benefit for the art therapy group apparent.

Interpretation of the positive significant result obtained on the SANS is difficult. On the one hand it would seem not unlikely that a form of group therapy might promote better social functioning of the kind that is assessed by some of the items on this measure. On the other hand, comparisons on other measures more explicitly addressing social functioning (the SFS and IIP32) did not yield significant differences in the planned analyses though there is a tendency for the SFS to improve more in the AT than the SPC group. In addition, pretreatment differences on the SANS complicate interpretation since there was a small but statistically significant difference between the AT and SPC arms at baseline on the SANS, with the AT group having lower scores at baseline. Despite the ANCOVA analysis taking baseline score into account, there can still be ways in which baseline differences can contribute to ANCOVA change differences. Nevertheless it should be noted that the control

Table I. Designed analysis results for comparison of the two trial arms.

\begin{tabular}{|c|c|c|c|c|c|c|}
\hline \multirow[b]{2}{*}{ Measure } & \multicolumn{3}{|c|}{ End of therapy } & \multicolumn{3}{|c|}{ Six month follow-up } \\
\hline & $\mathrm{F}$ & $\mathrm{df}$ & $p$ & $\mathrm{~F}$ & $\mathrm{df}$ & $p$ \\
\hline Perc QoL & 0.08 & 1,71 & .78 & 2.1 & 1,37 & .15 \\
\hline SFS & 1.7 & 1,66 & .20 & 1.1 & 1,36 & .30 \\
\hline BPRS & 2.6 & 1,70 & .11 & 2.3 & 1,37 & .14 \\
\hline HoNOS & 1.4 & 1,70 & .24 & 1.1 & 1,37 & .30 \\
\hline SANS & 5.5 & 1,70 & .02 & 5.3 & 1,37 & .03 \\
\hline IIP & 0.75 & 1,67 & .39 & 0.87 & 1,35 & .36 \\
\hline BSI & 0.93 & 1,62 & .34 & 1.3 & 1,34 & .25 \\
\hline
\end{tabular}




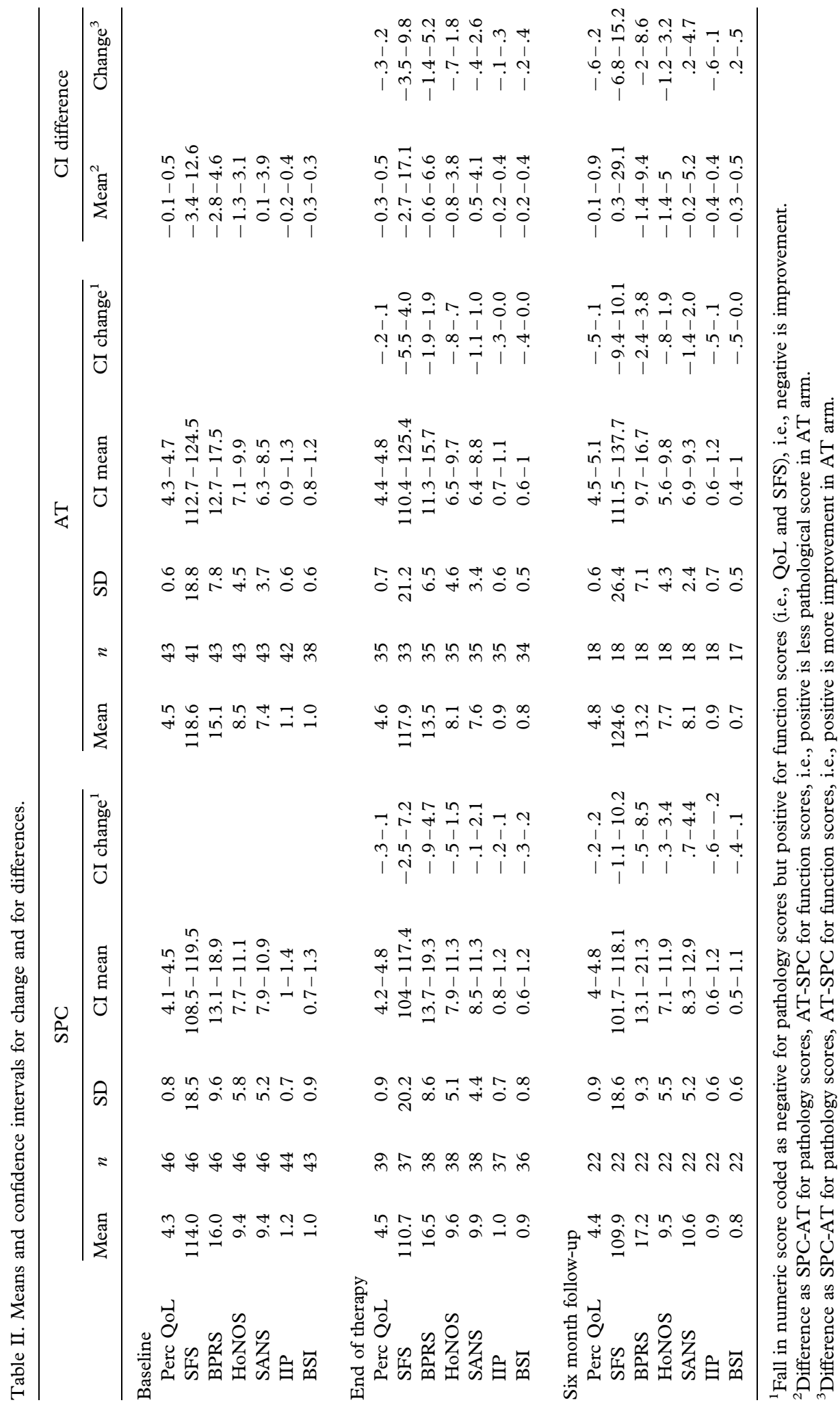


group deteriorated further at post-treatment where the AT group showed a slight improvement. This pattern seems more consistent with the occurrence of a true therapeutic effect than that of a statistical artefact.

Nevertheless it should be noted that as a consequence of recruitment difficulties, the trial was underpowered. Instead of recruiting $60+$ patients to each arm of the trial it was only possible to recruit approximately 45. Loss to follow-up further reduced this to around 20 in each arm by the six-month follow-up point. There was a tendency for the AT arm to improve more than the controls hence it is perfectly possible that an adequate sample size would have yielded a greater number of post-treatment comparisons showing significant differences.

It is also arguable that a 12-session treatment for a group of chronically handicapped individuals such as those in the present trial might itself be considered "underpowered". The majority of art therapists working with such populations would probably argue that it was and find it surprising that the results were as positive as they appear to be. Unfortunately resource constraints on the present study made it impossible to test a more substantial AT treatment, though clearly this would be a desirable research development.

In conclusion, the study has demonstrated the considerable logistic difficulties of achieving high levels of recruitment and follow-up with such populations. Though the recruitment and follow-up severely restricted the statistical power of the study and challenge generalizability, the proportion of eligible patients entering the study is still comparable with those entering pharmacological trials (Haberfellner, 2000; Yastrubetskaya, Chiu \& O'Connell, 1997). These results provide very tentative support for Art Therapy interventions with adult psychiatric patients with severe and/or complex problems. They also indicate the clear need for larger RCTs ideally involving somewhat longer periods of AT in both group and/or individual sessions: i.e., more representative of current clinical practice where AT is available for such patients, and for much more assessment of change in routine practice (Margison, Barkham, Evans, McGrath, Mellor-Clark, Audin, \& Connell, 2000).

\section{Clinical implications}

- Group art therapy was acceptable to adult psychiatric patients with severe and/or complex problems.

- Such therapy lasting only 12 weeks had a statistically significant impact on negative symptoms though little and non-significant impact on other measures.

\section{Limitations}

- Funding restrictions mean that the therapy was shorter than most art therapists would wish to offer such a client group.

- Funding and recruitment difficulties resulted in the study being statistically underpowered.

- Health economic measures and service utilization should be explored in further studies of art therapy with such patients.

\section{References}

Andreason, N. C. (1989). The Scale for the Assessment of Negative Symptoms (SANS): Conceptual and theoretical foundations. British fournal of Psychiatry, 155 (Suppl. 7), 49-52.

Barkham, M., Hardy, G. E., \& Startup, M. (1995). The IIP-32: A short version of the Inventory of Interpersonal Problems. British fournal of Clinical Psychology, 35, 21-35. 
Birchwood, M., Smith, J., Cochrane, R., Wetton, S., \& Copestake, S. (1990). The Social Functioning Scale. British Fournal of Psychiatry, 157, 853-859.

Borchers, K. K. (1985). Do gains made in group art therapy persist? A study with aftercare patients. American Fournal of Art Therapy, 23, 89-91.

Derogatis, L. R., \& Melisaratos, N. (1983). The Brief Symptom Inventory: An introductory report. Psychological Medicine, 13, 595-605.

Faul, F., \& Erdfelder, E. (1992). GPOWER: A priori, post-hoc, and compromise power analyses for MS-DOS [Computer program]. Bonn, FRG: Bonn University, Dept of Psychology.

Green, B. L., Wehling, C., \& Talsky, G. J. (1987). Group art therapy as an adjunct to treatment for chronic outpatients. Hospital $\mathcal{G}$ Community Psychiatry, 38, 988-991.

Grodner, S., Braff, D. L., Janowsky, D. S., \& Clopton, P. L. (1982). Efficacy of art/movement therapy in elevating mood. Arts in Psychotherapy, 9, 217-225.

Haberfellner, E. M. (2000). Recruitment of depressive patients for a controlled clinical trial in a psychiatric practice. Pharmacopsychiatry, 33, $142-144$.

Margison, F. R., Barkham, M., Evans, C., McGrath, G., Mellor-Clark, J., Audin, K., \& Connell, J. (2000). Measurement and psychotherapy: Evidence-based practice and practice-based evidence. British fournal of Psychiatry, 177, 123-130.

Moher, D., Schulz, K. F., \& Altman, D. for the CONSORT Group (Consolidated Standards of Reporting Trials). (2001). The CONSORT statement: Revised recommendations for improving the quality of reports of parallel group randomised trials. Fournal of the American Medical Association, 285(15), 1987-1991.

Oliver, J., Huxley, P., Bridges, K., \& Hadi, M. (1996). Quality of life and mental health services. Florence, KY: Taylor and Francis.

Overall, J. E., \& Gorham, D. R. (1962). The Brief Psychiatric Rating Scale. Psychological Reports, 10, 799-812.

Pocock, S. J. (1984). Clinical trials: A practical approach. Chichester: Wiley.

Potocky, M. (1993). An Art Therapy Group for clients with chronic schizophrenia. Social Work with Groups, 16, $73-82$.

Ruddy, R., \& Milnes, D. (2003). Art therapy for schizophrenia or schizophrenia-like illnesses. Cochrane Library.

Saunders, E. J., \& Saunders, J. A. (2000). Evaluating the effectiveness of art therapy through a quantitative outcomes-focussed study. The Arts in Psychotherapy, 27, 99-106.

Schut, H. A. W., de Keijser, J., van den Bout, J., et al. (1996). Cross-modality group therapy: Description and assessment of a new program. Fournal of Clinical Psychology, 52, 357-365.

Tibbetts, T. J., \& Stone, B. (1990). Short-term art therapy with seriously emotionally disturbed adolescents. Special Issue: The creative arts therapies with adolescents. Arts in Psychotherapy, 17, 139-146.

Waller, D. (1993). Group interactive art therapy. London: Routledge.

Waller, D., \& Gilroy, A. (1992). Art therapy: $A$ handbook. London: Routledge.

Wing, J., Beevor, A., Curtis, R., Park, S., Hadden, S., \& Burns, A. (1998). Health of the Nation Outcome Scales (HoNOS). Research and development. British fournal of Psychiatry, 172, 11-18.

Yastrubetskaya, O., Chiu, E., \& O'Connell, S. (1997). "Is good clinical research practice for clinical trials good clinical practice?" International fournal of Geriatric Psychiatry, 12, 227-231. 